\title{
Efficacy of thoracoscopic surgery in the treatment of lung cancer in the perioperative period and its effects on serum D-dimer
}

\author{
YUANSHAN YAO, HAIBO SHEN, YINJIE ZHOU, ZHENHUA YANG and HONGBO HUANG \\ Department of Thoracic Surgery, Ningbo No. 2 Hospital, Haishu, Ningbo, Zhejiang 315000, P.R. China
}

Received August 29, 2017; Accepted January 4, 2018

DOI: $10.3892 / \mathrm{ol} .2018 .7839$

\begin{abstract}
The aim of this study was to investigate the feasibility and safety of thoracoscopic surgery in the treatment of lung cancer and its effect on serum D-dimer. A total of 218 patients with lung cancer treated in the Department of Thoracic Surgery of Ningbo No. 2 Hospital from January 1, 2013 to December 31, 2016, were retrospectively analyzed. Of the 218 patients, 120 patients underwent thoracotomy (thoracotomy group) and 98 patients underwent thoracoscopic surgery (thoracoscopy group). The clinical efficacy in the perioperative period and serum D-dimer level were compared between the two groups. In the present study, the intraoperative blood loss, blood transfusion rate, postoperative hospital stay, thoracic drainage time and volume in the thoracoscopy group were significantly shorter or smaller than those in the thoracotomy group $(\mathrm{P}<0.05)$, but there was no statistically significant difference in the average operation time between the thoracoscopy and the thoracotomy group. The incidence rate from moderate to severe pains in incisions after operation, the use rate of analgesics and the average disappearance time of the pain in incisions in the thoracoscopy were lower than those in the thoracotomy group $(\mathrm{P}<0.05)$. The amount of serum $\mathrm{D}$-dimer immediately after operation in the thoracotomy group was significantly increased compared with that before operation $(\mathrm{P}<0.05)$, but there was no significant increase in the thoracoscopy group. At $24 \mathrm{~h}$ after operation, the serum D-dimer level in the two groups was further increased $(\mathrm{P}<0.05)$, and the comparison between the two groups showed that the levels of serum D-dimer in the thoracoscopy group immediately and at $24 \mathrm{~h}$ after operation were significantly lower than those in the thoracotomy group $(\mathrm{P}<0.05)$. The incidence rate of postoperative complications in the thoracoscopy was lower than that in the thoracotomy group, but the difference was not statistically significant. Our results show that thoracoscopic surgery is feasible and safe in
\end{abstract}

Correspondence to: Dr Haibo Shen, Department of Thoracic Surgery, Ningbo No. 2 Hospital, 41 Xibei Street, Haishu, Ningbo, Zhejiang 315000, P.R. China

E-mail: abeew4@163.com; shbfly7516@163.com

Key words: thoracoscopy, lung cancer, perioperative period, serum D-dimer, clinical efficacy the treatment of lung cancer. Compared with the thoracotomy group, the intraoperative condition and postoperative recovery have obvious advantages. The postoperative blood of patients is hypercoagulable and D-dimer increased gradually after $24 \mathrm{~h}$. The effect of thoracoscopic surgery on serum D-dimer is relatively less effective.

\section{Introduction}

Lung cancer is a common malignant tumor, whose incidence and mortality rate show sharp upward trends $(1,2)$. In China, morbidity and mortality rates of lung cancer exhibit an obvious increasing trend. Lung cancer has become the leading cause of mortality compared to other cancer types, and the mortality rate has been increased by $75.77 \%$ compared with that in the 1990s (3).

In previous years, with the continuous development of thoracoscopic surgery techniques and equipment, thoracoscopic surgery for the radical treatment of lung cancer has drawn increasing attention from clinicians. Thoracoscopic surgery has been widely used in clinical treatment because of small operation wound and rapid postoperative recovery, which provides surgical opportunity for elderly patients with poor cardiopulmonary function (4). Previous findings showed that plasma D-dimer is the sign of hypercoagulability and fiber coagulation hyperfunction state. It also has important significance for clinical diagnosis, prognosis and staging of lung cancer $(5,6)$. In addition, researchers have begun to focus on the relationship between serum D-dimer level and different surgical methods (thoracoscopy or thoracotomy) (7). In the present study, the feasibility and safety of thoracoscopic surgery for lung cancer were evaluated by retrospective analyses, comparisons of intraoperative and postoperative cases of thoracoscopic surgery and traditional thoracotomy for lung cancer.

\section{Materials and methods}

General materials. Patients with lung cancer treated in the Department of Thoracic Surgery of Ningbo No. 2 Hospital (Zhejiang, China) from January 1, 2013 to December 31, 2016, were retrospectively analyzed. The study was approved by the Ethics Committee of Ningbo No. 2 Hospital and informed consents was signed by the patients. Inclusion criteria for the study were: i) Patients receiving radical surgery for lung cancer and pathologically diagnosed with lung cancer. 
A

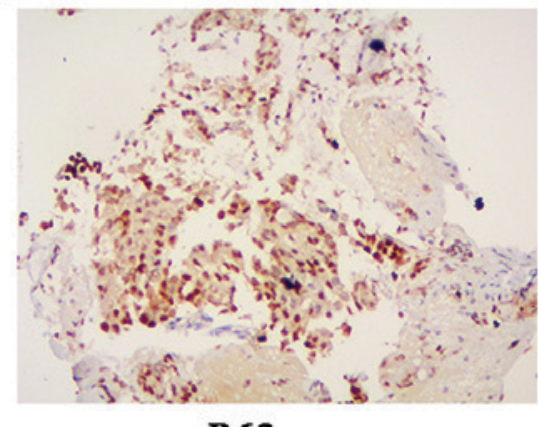

P63
B

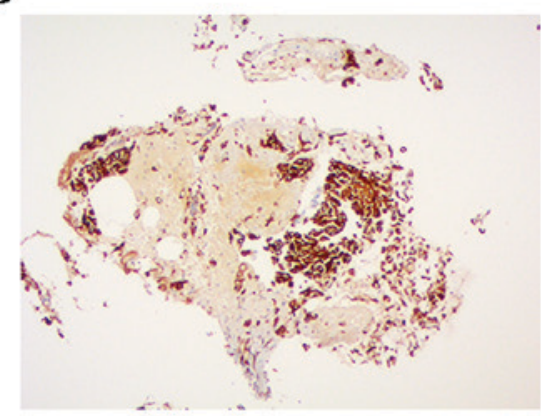

CK7
C

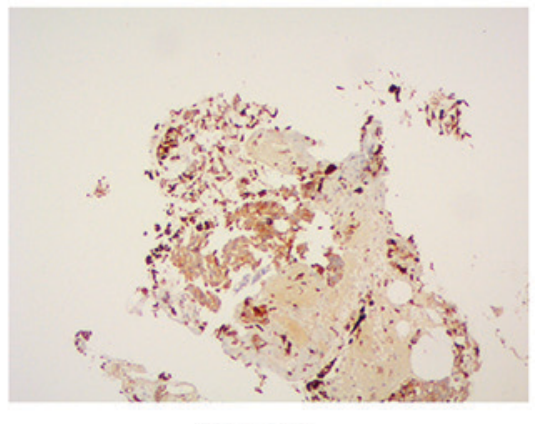

CD56

Figure 1. Immunohistochemical images for: (A) Lung squamous cell carcinoma, (B) adenocarcinoma, and (C) small cell carcinoma.

Immunohistochemical images for lung squamous cell carcinoma, adenocarcinoma and small cell carcinoma are shown in Fig. 1A-C, respectively; ii) male or female patients aged 18-70 years; iii) patients whose heart, brain, lung, kidney and other visceral organs functions were well before operation, and who could tolerate operations; iv) patients with no malignant arrhythmia, thyroid dysfunction or past histories; v) patients receiving thoracoscopic surgery who would not choose thoracotomy at the midterm of thoracoscopic surgery or change surgical methods during operation; and vi) patients who did not take anticoagulants recently. A total of 218 patients were included in the study, and divided into the thoracoscopy $(n=98)$ and thoracotomy $(n=120)$ groups according to the different surgical methods. The baseline data of the two groups of patients were compared, and the differences were not statistically significant $(\mathrm{P}>0.05)$ (Table I).

\section{Methods}

Preoperative preparations. Two groups of patients were treated with the same preoperative preparations, including respiratory exercise, smoking cessation, aerosol inhalation, correction of water, and electrolyte disorders. All the patients underwent preoperative computed tomography (CT), chest-enhanced CT and other tests for the confirmation of the diagnosis and stage. Mediastinoscopy and biopsy were not taken as routine examinations. Reserves and the compensatory capacity of the heart, lung, liver and kidney and the preserved skin of all the patients were comprehensively understood and assessed prior to surgery. In addition, before operation, gastrointestinal decompression tube and ureter were indwelt, and broad-spectrum antibiotics were retentively used. After the adequate preparation, surgical treatment was conducted.

Thoracoscopy group. Patients were placed in the lateral position, and were generally anesthetized through double-lumen endotracheal intubation. Then patients received one-lung ventilation in the lateral recumbent position for radical surgery for lung cancer. The 3-hole method was used for operations: The observation hole with the diameter of $15 \mathrm{~mm}$ was located at the 7th and 8th intercostal of the midaxillary line of patients, and the Strykerl088i-30 thoracoscopy was inserted. An operation mouth with the length of $30-50 \mathrm{~mm}$ was made at the 4th and 5th intercostal of the anterior axillary line, and a deputy operation mouth with the length of about $20 \mathrm{~mm}$ was made at the 6th and 7 th intercostal of the posterior axillary line or the 8th intercostal of the infrascapular line. Ribs of patients in the thoracoscopy group were not cut, and not distracted by a distractor. The cut lung lobes were placed in the specimen bag and removed from the operation mouth to protect the incision from the implantation metastasis and wound infection. Routine mediastinal lymphadenectomy in the hilus of the lung was conducted. At the end of the operation, the patients were monitored and treated in the intensive care unit, a breathing machine was used to assist breathing, and vital signs were dynamically monitored. The trachea cannulas were removed under the condition that patients woke up with normal vital signs and blood gas analysis results. Analgesia pumps were not routinely used after operation.

Thoracotomy group. Radical surgery for lung cancer was completed by accessing the chest through conventional posterior incision.

D-dimer method. Patients were examined by extracting peripheral blood preoperatively, immediately and $24 \mathrm{~h}$ after surgery. The blood was preserved in a citrate anticoagulant vacuum tube for parallel plasma $\mathrm{D}$-dimer determination.

Observation indexes. The operation time, intraoperative blood loss, blood transfusion, postoperative thoracic drainage time and volume, postoperative hospital stay, the incidence rate from moderate to severe pains in incisions at $6 \mathrm{~h}$ after operation (FACES ${ }^{\circledR}$ Pain Rating Scale), the use rate of analgesics, the time of the disappearance of pain in incisions after operation and relevant postoperative complications within the perioperative period of patients were observed. In addition, levels of the serum D-dimer in the two groups of patients before operation, immediately after operation and at $24 \mathrm{~h}$ after operation were observed. The change of prothrombin time (PT), activated partial thromboplastin time (APTT), thrombin time (TT), blood coagulation factor V and VII of patients before and after surgery were also observed and recorded.

Statistical analysis. SPSS 17.0 (SPSS, Inc., Chicago, IL, USA) statistical software was used for analysis. Measurement data were expressed as mean \pm SD. Intergroup comparisons were detected using the t-test, and comparisons among multiple 
Table I. Comparisons of clinical data of patients between the thoracotomy and the thoracoscopy groups.

\begin{tabular}{|c|c|c|c|c|}
\hline General data & $\begin{array}{l}\text { Thoracotomy group } \\
\qquad(\mathrm{n}=120)\end{array}$ & $\begin{array}{l}\text { Thoracoscopy group } \\
\qquad(\mathrm{n}=98)\end{array}$ & $\chi^{2} / \mathrm{t} / \mathrm{Z}$ & P-value \\
\hline Age (years) & $55.63 \pm 8.01$ & $56.04 \pm 6.92$ & 0.792 & 0.473 \\
\hline \multicolumn{5}{|l|}{ Sex } \\
\hline $\mathrm{M} / \mathrm{F}$ & $68 / 52$ & $52 / 46$ & 0.280 & 0.590 \\
\hline \multicolumn{5}{|l|}{ Clinical symptoms, n (\%) } \\
\hline Cough & $53(44.17)$ & $45(45.92)$ & 0.067 & 0.796 \\
\hline Expectoration & $20(16.67)$ & $10(10.20)$ & 2.730 & 0.099 \\
\hline Hemoptysis & $32(26.67)$ & $24(24.49)$ & 0.134 & 0.714 \\
\hline Chest distress & $12(10.00)$ & $7(7.14)$ & 0.554 & 0.457 \\
\hline Emaciation & $33(27.50)$ & $28(28.57)$ & 0.031 & 0.861 \\
\hline Pyrexia & $17(14.17)$ & $11(11.22)$ & 0.417 & 0.518 \\
\hline No symptom & $20(16.67)$ & $16(16.33)$ & 0.005 & 0.946 \\
\hline \multicolumn{5}{|l|}{ Past history, n (\%) } \\
\hline Smoking & $82(68.33)$ & $60(61.22)$ & 1.200 & 0.273 \\
\hline Hypertension & $23(19.17)$ & $16(16.33)$ & 0.296 & 0.586 \\
\hline Diabetes & $32(26.67)$ & $21(21.43)$ & 0.804 & 0.370 \\
\hline COPD & $34(28.33)$ & $19(19.39)$ & 2.346 & 0.126 \\
\hline \multicolumn{5}{|l|}{ The ASA grading, n (\%) } \\
\hline Grade I & $42(35.00)$ & $45(45.92)$ & & \\
\hline Grade II & $65(54.17)$ & $45(45.92)$ & & \\
\hline Grade III & $13(10.83)$ & $8(8.16)$ & & \\
\hline Maximum diameter of lesion (cm) & $2.81 \pm 0.32$ & $3.05 \pm 0.50$ & 0.469 & 0.674 \\
\hline Lesion site, n (\%) & & & -0.723 & 0.470 \\
\hline Left upper lobe & $28(23.33)$ & $25(25.51)$ & & \\
\hline Left lower lobe & $16(13.33)$ & $15(15.31)$ & & \\
\hline Right upper lobe & $28(23.33)$ & $23(23.47)$ & & \\
\hline Right middle lobe & $12(10.00)$ & $10(10.20)$ & & \\
\hline Right lower lobe & $36(30.00)$ & $25(25.51)$ & & \\
\hline Pathological type, n (\%) & & & -1.903 & 0.057 \\
\hline Squamous carcinoma & $39(32.50)$ & $42(42.86)$ & & \\
\hline Adenocarcinoma & $46(38.33)$ & $38(38.78)$ & & \\
\hline Adeno-squamous carcinoma & $18(15.00)$ & $10(10.20)$ & & \\
\hline Others & $17(14.17)$ & $8(8.16)$ & & \\
\hline Pathological stage, n (\%) & & & -0.444 & 0.657 \\
\hline IA & 35 (29.17) & $31(31.63)$ & & \\
\hline IB & $42(35.00)$ & $35(35.71)$ & & \\
\hline IIA & $16(13.33)$ & $12(12.24)$ & & \\
\hline IIB & $17(14.17)$ & $10(10.20)$ & & \\
\hline IIIA & $9(7.50)$ & $10(10.20)$ & & \\
\hline IIIB & $1(0.83)$ & 0 & & \\
\hline Serum D-dimer (mg/l) & $0.37 \pm 0.16$ & $0.36 \pm 0.15$ & -0.148 & 0.882 \\
\hline
\end{tabular}

M/F, male/female; COPD, chronic obstructive pulmonary disease; ASA, American Society of Anesthesiologists.

groups or intragroup comparisons were detected using the analysis of variance. Countable data were expressed as a percentage or composition ratio, and intergroup comparisons were detected using the $\chi^{2}$ test. Ranked data were expressed as a percentage or composition ratio, and intergroup comparisons were detected using the rank-sum test. Inspection level was $\alpha=0.05$.

\section{Results}

Intraoperative condition. Operations of all the patients were successfully completed. The blood loss and transfusion rate in the thoracoscopy were significantly lower than those in the thoracotomy group $(\mathrm{P}<0.05)$, but there was no statistically 
Table II. Comparison of intraoperative condition of patients between the thoracotomy and thoracoscopy groups.

\begin{tabular}{lccrr}
\hline $\begin{array}{l}\text { Intraoperative } \\
\text { condition }\end{array}$ & $\begin{array}{c}\text { Thoracotomy } \\
\text { group }(\mathrm{n}=120)\end{array}$ & $\begin{array}{l}\text { Thoracoscopy } \\
\text { group }(\mathrm{n}=98)\end{array}$ & $\chi^{2 / \mathrm{t}}$ & P-value \\
\hline $\begin{array}{l}\text { Operative time (min) } \\
\text { Intraoperative }\end{array}$ & $126.17 \pm 39.23$ & $132.23 \pm 32.56$ & 1.106 & 0.276 \\
$\begin{array}{l}\text { blood loss (ml) } \\
\text { Blood transfusion } \mathrm{n}(\%)\end{array}$ & $234.23 \pm 53.72$ & $110.64 \pm 18.25$ & -6.316 & $<0.001$ \\
\hline
\end{tabular}

Table III. Comparison of postoperative recovery condition of patients between the thoracotomy and thoracoscopy groups.

\begin{tabular}{lcccr}
\hline Postoperative condition & $\begin{array}{c}\text { Thoracotomy group } \\
(\mathrm{n}=120)\end{array}$ & $\begin{array}{c}\text { Thoracoscopy group } \\
(\mathrm{n}=98)\end{array}$ & $\chi^{2} / \mathrm{t}$ & P-value \\
\hline Thoracic drainage time (days) & $6.27 \pm 2.13$ & $4.38 \pm 1.92$ & -5.670 & $<0.001$ \\
Thoracic drainage volume (ml) & $1453.57 \pm 611.25$ & $1024.78 \pm 556.95$ & -5.743 & $<0.001$ \\
Postoperative hospital stay (days) & $13.84 \pm 2.31$ & $10.12 \pm 2.56$ & -5.694 & $<0.001$ \\
$\begin{array}{l}\text { Incidence rate of moderate to severe pains } \\
\text { in incisions at 6 h after operation, } \mathrm{n}(\%)\end{array}$ & $72(60.00)$ & $20(20.41)$ & 34.667 & $<0.001$ \\
$\begin{array}{l}\text { Use rate of analgesics, } \mathrm{n}(\%) \\
\text { Average time of the }\end{array}$ & $68(56.67)$ & $18(18.37)$ & 33.126 & $<0.001$ \\
disappearance of pain in incisions (h) & $72.58 \pm 20.98$ & $28.67 \pm 14.37$ & -53.642 & $<0.001$ \\
\hline
\end{tabular}

Table IV. Comparison of changes in serum D-dimer of patients after operation between the thoracotomy and thoracoscopy groups $(\mathrm{mg} / \mathrm{l})$.

\begin{tabular}{|c|c|c|c|c|}
\hline Serum D-dimer & $\begin{array}{l}\text { Thoracotomy group } \\
\qquad(\mathrm{n}=120)\end{array}$ & $\begin{array}{l}\text { Thoracoscopy group } \\
\qquad(\mathrm{n}=98)\end{array}$ & $\mathrm{t}$ & P-value \\
\hline Before operation & $0.37 \pm 0.16$ & $0.36 \pm 0.15$ & -0.148 & 0.882 \\
\hline Immediately after operation & $0.55 \pm 0.26$ & $0.42 \pm 0.19$ & 4.001 & $<0.001$ \\
\hline At $24 \mathrm{~h}$ after operation & $0.93 \pm 0.17$ & $0.68 \pm 0.23$ & 8.585 & $<0.001$ \\
\hline F-value & 277.785 & 67.492 & & \\
\hline P-value & $<0.001$ & $<0.001$ & & \\
\hline $\mathrm{P} 1: 2$ & $<0.001$ & 0.132 & & \\
\hline $\mathrm{P} 1: 3$ & $<0.001$ & $<0.001$ & & \\
\hline $\mathrm{P} 2: 3$ & $<0.001$ & $<0.001$ & & \\
\hline
\end{tabular}

significant difference in the average operation time between the thoracoscopy and thoracotomy groups (Table II).

\section{Postoperative condition}

Postoperative recovery condition. The postoperative hospital stay, the thoracic drainage time and volume in the thoracoscopy group were shorter or smaller than those in the thoracotomy group $(\mathrm{P}<0.05)$ (Table III). No pain pump was used in both groups. Postoperative pain was assessed by the FACES ${ }^{\circledR}$ Pain Rating Scale at $6 \mathrm{~h}$ after operation. The incidence rate of moderate to severe pain in incisions, the use rate of analgesics and the average time of the disappearance of pain in incisions in the thoracoscopy were lower than those in the thoracotomy group $(\mathrm{P}<0.05)$ (Table III).
Changes in serum D-dimer after operation. The level of serum D-dimer in the two groups after operation was significantly higher than that before operation, and the serum D-dimer level of patients was further increased at $24 \mathrm{~h}$ after operation $(\mathrm{P}<0.05)$. The comparison between the two groups showed that the levels of serum D-dimer in the thoracoscopy group immediately and at $24 \mathrm{~h}$ after operation were significantly lower than those in the thoracotomy group $(\mathrm{P}<0.05)$ (Table IV and Fig. 2). There was no significant difference of PT, APTT and TT in the two groups before and after surgery $(\mathrm{P}>0.05)$. Coagulation factor $\mathrm{V}$ and VII were elevated after surgery compared with those before surgery $(\mathrm{P}<0.05)$. No significant differences were identified between the two groups $(\mathrm{P}>0.05)$ (Table V). 
Table V. The changes of blood coagulation indexes before and after operation of the two groups (mean \pm SD).

\begin{tabular}{llccccc}
\hline \multirow{2}{*}{ Groups } & Time & PT $(\mathrm{sec})$ & APTT $(\mathrm{sec})$ & TT $(\mathrm{sec})$ & $\begin{array}{c}\text { Coagulation } \\
\text { factor V }(\mathrm{g} / \mathrm{l})\end{array}$ & $\begin{array}{c}\text { Coagulation } \\
\text { factor VII }(\mathrm{g} / \mathrm{l})\end{array}$ \\
\hline Thoracoscopy & Before surgery & $12.32 \pm 0.87$ & $28.65 \pm 2.65$ & $17.32 \pm 2.34$ & $2.19 \pm 0.43$ & $2.19 \pm 0.43$ \\
& After surgery & $12.88 \pm 0.88$ & $31.22 \pm 2.12$ & $18.32 \pm 3.02$ & $1.81 \pm 0.32^{\mathrm{a}}$ & $1.81 \pm 0.32^{\mathrm{a}}$ \\
Thoracotomy & Before surgery & $12.45 \pm 0.98$ & $28.98 \pm 2.01$ & $17.43 \pm 2.19$ & $2.23 \pm 0.52$ & $2.23 \pm 0.52$ \\
& After surgery & $12.78 \pm 0.97$ & $32.43 \pm 1.35$ & $18.97 \pm 3.21$ & $1.82 \pm 0.54^{\mathrm{a}}$ & $1.82 \pm 0.54^{\mathrm{a}}$ \\
\hline
\end{tabular}

Compared with that before surgery; ${ }^{\mathrm{a}} \mathrm{P}<0.05 ; \mathrm{SD}$, standard deviation; PT, prothrombin time; APTT, activated partial thromboplastin time; TT, thrombin time.

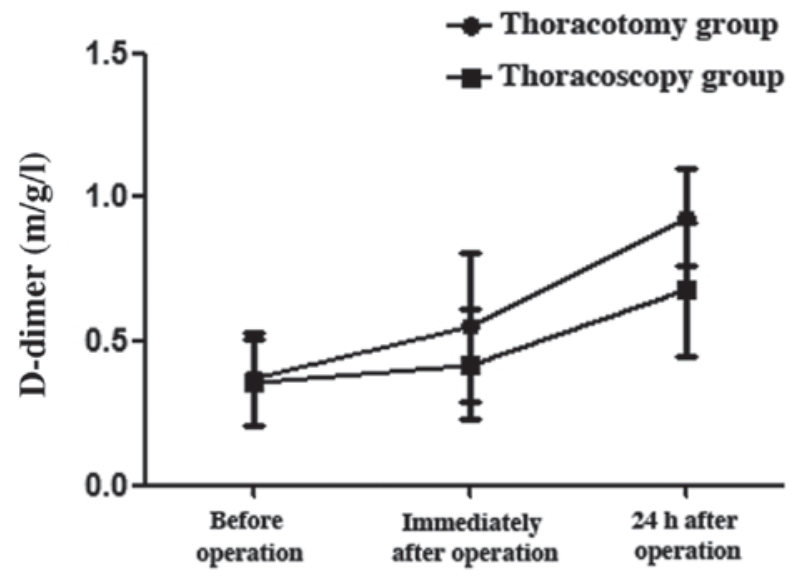

Figure 2. Comparison of serum D-dimer level of patients after operation between the thoracotomy and thoracoscopy groups. The D-dimer content of the two groups is gradually increased with the increase of time, and D-dimer content in the thoracoscopy group is lower than that in the thoracotomy group at the same time point.

Postoperative complications. Although the total incidence rate of postoperative complications in the thoracoscopy was lower than that in the thoracotomy group, there was no statistically significant difference between the two groups. Only the incidence rate of wound infection in the thoracoscopy was significantly lower than that in the thoracotomy group $(\mathrm{P}<0.05)$ (Table VI). Among the patients, there were 28 patients with arrhythmia, which was improved after observation or through treatment with antiarrhythmic drugs; there were 3 patients with atelectasis, which was improved through active expectoration, anti-infection, bronchoscopy suction and other treatments; there were 46 patients with pulmonary infection, which was improved by active expectoration and anti-infection treatments; there were 4 patients with air leak at 5 days after operation, which was stopped by the intrapleural injection of albumins and other treatments; there were 3 patients with empyema, which was improved by receiving thoracic close drainage, rinse, nutrition support and anti-infection treatments; there was 1 patient with thoracic hemorrhage and 1 patient with chylothorax, who were treated with thoracotomy again; and there were 12 patients with wound infection due to obesity and the history of diabetes, who were discharged after the wound dressing was changed and anti-infection treatment was conducted (Table V).

\section{Discussion}

Since the 1990s, video-assisted thoracoscopic surgery has been increasingly widely used in the treatment of benign and malignant pulmonary diseases. Compared with traditional thoracotomy, thoracoscopic surgery is characterized by minimal trauma, mild postoperative pain, fewer postoperative complications, short hospital stay, good aesthetic appearance and other advantages (8). However, a study has shown that thoracoscopic surgery has no major advantages compared with traditional thoracotomy, but has a long operation time and may increase intraoperative complications (9). Kuritzky and $\mathrm{Ng}$ (10) found that no significant difference was shown regarding the curative effect in the treatment of elderly patients with non-small cell lung cancer between thoracoscopic lung surgery and thoracotomy. Nevertheless, the amount of bleeding and complication conditions are better in thoracoscopic lung surgery with shorter postoperative hospitalization time and lighter early inflammatory reaction. Nwogu et al (11) suggested that full thoracoscopical lung resection of lymph node dissection in the treatment of peripheral lung cancer is safe, effective, and feasible with less postoperative pleural fluid. It can achieve the effect of conventional thoracic surgery in terms of radical lymph node dissection. Gopaldas et al (12) showed that the single hole thoracoscopic lobectomy for the treatment of early peripheral lung cancer is a safe and effective operation.

In the present study, the blood loss and transfusion rate in the thoracoscopy were less than those in the thoracotomy group, but the average operation time and postoperative complications in the two groups were equivalent. This was mainly because when the retrospectively analyzed patients were included, the thoracoscopic surgery techniques of the Department of Thoracic Surgery of our hospital has become relatively more mature, and clinicians were relatively more experienced, which obviously made the thoracoscopic surgery more safe and reliable than that before the techniques were advanced. In thoracoscopic surgery, the field of vision is wide and clear, the anatomical structure is clear, the incision is small and the errhysis volume is small, thus leading to little intraoperative blood loss in thoracoscopic surgery. A prospective study of Flores et al (13) showed that the number of patients with postoperative complications, including atrial fibrillation, persistent pulmonary air leak, atelectasis, and pneumonia, in the thoracoscopy was significantly less than that in the thoracotomy group. However, in the present study, 
Table VI. Comparison of condition of postoperative complications of patients between the thoracotomy and thoracoscopy groups [n (\%)].

\begin{tabular}{lcccc}
\hline Complication & $\begin{array}{c}\text { Thoracotomy group } \\
(\mathrm{n}=120)\end{array}$ & $\begin{array}{c}\text { Thoracoscopy group } \\
(\mathrm{n}=98)\end{array}$ & $\chi^{2}$ & P-value \\
\hline Arrhythmia & $17(14.17)$ & $11(11.22)$ & 0.417 & 0.518 \\
Atrial fibrillation & $10(8.33)$ & $6(6.12)$ & 0.388 & 0.533 \\
Supraventricular tachycardia & $4(3.33)$ & $5(5.10)$ & 0.055 & 0.815 \\
Ventricular premature beat & $1(0.83)$ & 0 & 0.000 & 1.000 \\
Ventricular fibrillation & 0 & 0 & 0.000 & 1.000 \\
Sinus bradycardia & $2(1.67)$ & 0 & 0.325 & 0.569 \\
Lung & $1(0.83)$ & & & \\
Atelectasis & $26(21.67)$ & $2(2.04)$ & 0.031 & 0.860 \\
Pulmonary infection & $2(1.67)$ & $20(20.41)$ & 0.051 & 0.821 \\
Pulmonary air leak after operation & $2(1.67)$ & $2(2.04)$ & 0.000 & 1.000 \\
Empyema & $1(0.83)$ & $1(1.02)$ & 0.000 & 1.000 \\
Chylothorax & 0 & 0 & 0.000 & 1.000 \\
Thoracic hemorrhage & $10(8.33)$ & $1(1.02)$ & 0.000 & 1.000 \\
Incision infection & $3(2.50)$ & $2(2.04)$ & 4.106 & 0.043 \\
Deep venous thrombosis & $62(51.67)$ & 0 & 0.984 & 0.321 \\
Total & & $39(39.80)$ & 3.057 & 0.080 \\
\hline
\end{tabular}

the incidence rate of postoperative complications in the two groups was equivalent, and only the incidence rate of wound infection in the thoracoscopy was significantly lower than that in the thoracotomy group. In our study, complications in the thoracoscopy group were mainly distributed in pulmonary infection and arrhythmia. Pulmonary infection was mainly due to the history of smoking and pulmonary diseases in recent years, while preoperative and postoperative banning on smoking increased the psychological burden of patients. A study has revealed that more than 400 cigarettes a year, combined with basic pulmonary diseases and the forced expiratory volume in one second (FEV1\%) less than or equal to $60 \%$ are high-risk factors for the incidence of complications of lung cancer surgeries (14). At present, it is still controversial whether the incidence rate of postoperative arrhythmia in patients with lung cancer is affected by thoracoscopic surgery or the traditional thoracotomy method. Arrhythmia is a common complication of thoracic tumor surgery, commonly manifesting as atrial fibrillation, and its incidence rate is more than $20 \%(15,16)$. The results of the present study indicated that the number of patients with arrhythmia including atrial fibrillation in the thoracoscopy group was not significantly reduced compared with that in the conventional thoracotomy group. This result is similar to that obtained in the study of Park et al (17). However, a study revealed that the incidence rate of arrhythmia in the pulmonary lobectomy under the thoracoscopy is significantly lower than that in the conventional thoracotomy (18).

$\mathrm{D}$-dimer is the product degraded from fibrin polymers under the action of plasmin after fibrin monomers form fibrin polymers through the crosslinking of the activation factor, XIII, and it is a specific index embodying human secondary fibrinolysis hyperthyroidism (19). Surgical injury and anesthetic stimulation can lead to increased levels of prothrombin fragment, fibrinopeptide A and other substances, which can alter the coagulation status of the body. In research by Allaix et al (20), suspected VTE patients with negative D-dimer were followed up for 6 months. Thrombosis occurred in only $1.9 \%$ of patients. VTE patients with a high level of D-dimer are usually recovered to a normal status in about a month. In the present study, the levels of serum D-dimer immediately and at $24 \mathrm{~h}$ after operation in the two groups were significantly higher than that before operation, and the change in serum D-dimer in the thoracotomy was more obvious than that in the thoracoscopy group. Since the trauma of thoracoscopic surgery is small, the incidence rate of postoperative hypercoagulable state of blood is lower than that of traditional thoracotomy, and similar results have been reported in many articles regarding the thoracoscopic and laparoscopic surgeries (21). Previous findings have shown that serum D-dimer level is increased in a variety of malignant tumors, and it is closely related to tumor stage, lymph node metastasis and other factors (22). Some studies have indicated that the increased level of serum D-dimer is significantly associated with poor prognosis of lung cancer $(6,19)$. Therefore, the main reason for the increase in serum D-dimer level in this study needs to be further studied.

With the continuous development of thoracoscopic surgery techniques and equipment, radical surgery for lung cancer using thoracoscopy has achieved good results. The advantages of thoracoscopic surgery in the perioperative period have become increasingly apparent, and its relatively reduced effect on the postoperative hypercoagulable state of blood has drawn increasing attention.

\section{Acknowledgements}

Not applicable. 


\section{Funding}

No funding was received.

\section{Availability of data and materials}

The datasets used and/or analyzed during the current study are available from the corresponding author on reasonable request.

\section{Authors' contributions}

YY interpreted the data and drafted this manuscript. HS conceived and designed this study. $\mathrm{YZ}$ and $\mathrm{HH}$ collected and analyzed the data. ZY finally revised and approved the manuscript. All authors read and approved the final manuscript

\section{Ethics approval and consent to participate}

The present study was approved by the Ethics Committee of Ningbo No. 2 Hospital. Patients who participated in this research, signed the informed consent.

\section{Consent for publication}

Not applicable.

\section{Competing interests}

The authors declare that they have no competing interests.

\section{References}

1. Torre LA, Bray F, Siegel RL, Ferlay J, Lortet-Tieulent J and Jemal A: Global cancer statistics, 2012. CA Cancer J Clin 65: 87-108, 2015.

2. Jemal A, Bray F, Center MM, Ferlay J, Ward E and Forman D: Global cancer statistics. CA Cancer J Clin 61: 69-90, 2011.

3. Chen WQ, Zhang SW and Zou XN: A study on the estimation and trend of death of lung cancer in China. Chin J Lung Cancer 13: 488-493, 2010 (In Chinese).

4. Nakamura H and Taniguchi Y: Robot-assisted thoracoscopic surgery: current status and prospects. Gen Thorac Cardiovasc Surg 61: 127-132, 2013.

5. Vandlac AA, Cowan NG, Chen Y, Anderson RE, Conlin MJ, La Rochelle JC, Amling CL and Koppie TM: Timing, incidence and risk factors for venous thromboembolism in patients undergoing radical cystectomy for malignancy: A case for extended duration pharmacological prophylaxis. J Urol 191: 943-947, 2014.

6. Zhang PP, Sun JW, Wang XY, Liu XM and Li K: Preoperative plasma D-dimer levels predict survival in patients with operable non-small cell lung cancer independently of venous thromboembolism. Eur J Surg Oncol 39 :951-956, 2013.

7. Qi Y and Fu J: Research on the coagulation function changes in non small cell lung cancer patients and analysis of their correlation with metastasis and survival. J BUON 22: 462-467, 2017.

8. Laursen LO, Petersen RH, Hansen HJ, Jensen TK, Ravn J and Konge L: Video-assisted thoracoscopic surgery lobectomy for lung cancer is associated with a lower 30-day morbidity compared with lobectomy by thoracotomy. Eur J Cardiothorac Surg 49: 870-875, 2016.
9. Spartalis E, Mantonakis E, Athanasiou A and Moris D: Lobectomy by video-assisted thoracic surgery or muscle-sparing thoracotomy for stage 1 lung cancer: Could cost-effectiveness give the answer? J Am Coll Surg 221: 890, 2015.

10. Kuritzky AM and Ng T: Video-assisted thoracic surgery vs muscle-sparing thoracotomy: Prioritizing randomized trial to assess complications and long-term survival over cost comparisons: In reply to Spartalis and colleagues. J Am Coll Surg 221: 890-891, 2015.

11. Nwogu CE, D'Cunha J, Pang H, Gu L, Wang X, Richards WG, Veit LJ, Demmy TL, Sugarbaker DJ, Kohman LJ, et al: Alliance for clinical trials in oncology: VATS lobectomy has better perioperative outcomes than open lobectomy: CALGB 31001, an ancillary analysis of CALGB 140202 (Alliance). Ann Thorac Surg 99: 399-405, 2015.

12. Gopaldas RR, Bakaeen FG, Dao TK, Walsh GL, Swisher SG and Chu D: Video-assisted thoracoscopic versus open thoracotomy lobectomy in a cohort of 13,619 patients. Ann Thorac Surg 89: 1563-1570, 2010.

13. Flores RM, Park BJ, Dycoco J, Aronova A, Hirth Y, Rizk NP, Bains M, Downey RJ and Rusch VW: Lobectomy by video-assisted thoracic surgery (VATS) versus thoracotomy for lung cancer. J Thorac Cardiovasc Surg 138: 11-18, 2009.

14. Suzuki M, Otsuji M, Baba M, Saitoh Y, Iizasa T, Shibuya K, Sekine Y, Yoshida S and Fujisawa T: Bronchopleural fistula after lung cancer surgery. Multivariate analysis of risk factors. J Cardiovasc Surg 43: 263-267, 2002.

15. Muranishi Y,Sonobe M,Menju T, Aoyama A,Chen-YoshikawaTF, Sato T and Date H: Atrial fibrillation after lung cancer surgery: Incidence, severity, and risk factors. Surg Today 47: 252-258, 2017.

16. Zhang L and Gao S: Systematic review and meta-analysis of atrial fibrillation Prophylaxis After Lung Surgery. J Cardiovasc Pharmacol 67: 351-357, 2016.

17. Park BJ, Zhang H, Rusch VW and Amar D: Video-assisted thoracic surgery does not reduce the incidence of postoperative atrial fibrillation after pulmonary lobectomy. $\mathrm{J}$ Thorac Cardiovasc Surg 133: 775-779, 2007.

18. Imperatori A, Mariscalco G, Riganti G, Rotolo N, Conti V and Dominioni L: Atrial fibrillation after pulmonary lobectomy for lung cancer affects long-term survival in a prospective single-center study. J Cardiothorac Surg 7: 4, 2012.

19. Ay C, Dunkler D, Pirker R, Thaler J, Quehenberger P, Wagner O, Zielinski C and Pabinger I: High D-dimer levels are associated with poor prognosis in cancer patients. Haematologica 97: 1158-1164, 2012.

20. Allaix ME, Krane MK, Zoccali M, Umanskiy K, Hurst R and Fichera A: Postoperative portomesenteric venous thrombosis: Lessons learned from 1,069 consecutive laparoscopic colorectal resections. World J Surg 38: 976-984, 2014.

21. Diao D, Zhu K, Wang Z, Cheng Y, Li K, Pei L and Dang C: Prognostic value of the D-dimer test in oesophageal cancer during the perioperative period. J Surg Oncol 108: 34-41, 2013.

22. Zhang PP, Sun JW, Wang XY, Liu XM and Li K: Preoperative plasma D-dimer levels predict survival in patients with operable non-small cell lung cancer independently of venous thromboembolism. Eur J Surg Oncol 39: 951-956, 2013.

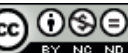

This work is licensed under a Creative Commons Attribution-NonCommercial-NoDerivatives 4.0 International (CC BY-NC-ND 4.0) License. 